\title{
Magnetoresistive Effect in PET Films with Iron Nanoparticles Synthesized by Ion Implantation
}

\author{
M.G. Lukashevich ${ }^{1}$, V.N. Popok ${ }^{2, *}$, V.S. Volobuev ${ }^{1}$, A.A. Melnikov ${ }^{1}$, R.I. Khaibullin ${ }^{3}$, \\ V.V. Bazarov ${ }^{3}$, A. Wieck ${ }^{4}$ and V.B. Odzhaev ${ }^{1}$ \\ ${ }^{1}$ Belarusian State University, Independence Ave. 4, 22050 Minsk, Belarus \\ ${ }^{2}$ University of Gothenburg, 42196 Gothenburg, Sweden \\ ${ }^{3}$ Kazan Physical-Technical Institute of RAS, Sibirsky Trakt 10/7, 420029 Kazan, Russia \\ ${ }^{4}$ Ruhr University, Universitatsstrasse 150, 44801 Bochum, Germany
}

\begin{abstract}
Thin polyethyleneterephthalate (PET) layers with Fe nanoparticles (NPs) were synthesized by high-fluence ion implantation. Temperature dependence of conductance and magnetoresistance, were studied as a function of ion fluence. It is found that the implantation with fluences of about $1.0 \times 10^{17} \mathrm{~cm}^{-2}$ causes high enough concentration of metal inclusions to provide conditions for electrical percolation that leads to an insulator-to-metal transition (IMT) in charge carrier transport mechanisms. The magnetoresistance measurements indicate that the magnetic percolation takes place at metal concentrations (fluences) lower than those needed for the electrical percolation. For the samples on insulating side of the IMT, a non-monotonous dependence of resistance in an increasing external magnetic field is observed due to anisotropic magnetoresistive effect and charge carrier scattering on magnetic inclusions. For the samples implanted with fluences $\geq$ $1.0 \times 10^{17} \mathrm{~cm}^{-2}$, the magnetoresistance becomes a monotonous decreasing function of the external magnetic field which is typical for ferromagnetic metals that indicates effective magnetic coupling of the iron inclusions.
\end{abstract}

Keywords: Polymers, Ion Implantation, Magnetic Nanoparticles, Magnetoresistance.

\section{INTRODUCTION}

High-fluence implantation of metal ions into polymers is a well-known way to embed metal species into dielectrics above a solubility limit and to synthesize media with metal nanoparticles (NPs) [1]. Depending on the metal species, such composite materials can be of interest for optical and magnetic applications [1,2]. However, one should consider the radiation damage effects that lead to modification of initial structure and properties of polymers $[3,4]$. In particular, electrical conductance of the implanted layer can increase up to values typical for semiconductors and even metals. This change in electrical properties is related to radiation-induced carbonization and formation of conjugated bonds [3-7]. In the presence of high metal concentration, which can reach some tens of at $\%$ in the implanted layer, there will be additional conductance mechanism due to the formation of metal inclusions. The situation becomes even more complicated if the implanted impurity is a magnetic metal. Spins of electrons start playing role and the conductance of such composite will vary in external magnetic field. On the one hand, the mechanisms of charge carrier transport can evolve from variable range hopping (VRH) or intercluster tunnelling for moderate metal concentration towards percolation conductance under the conditions of IMT [8]. On the other hand, an increase in the concentration of magnetic impurity in a nonmagnetic dielectric matrix leads to magnetic percolation transition from the diamagnetic to

*Address correspondence to this author at the University of Gothenburg, 42196 Gothenburg, Sweden; Tel: +46 31 7723435; Fax: +46 31 7723496; E-mail: popok@physics.gu.se ferromagnetic state through the superparamagnetism of magnetic NPs [9-11]. Moreover, granular condensed media with magnetic ordering can demonstrate a wide spectrum of different magnetic micro- or nano-structures. These structures are characterized by metastable states that complicate the understanding of charge carrier transport, in particular, due to possible scattering on domain walls [12]. These materials also become sensitive to magnetic history.

Thus, investigation of transport mechanisms in nanostructured magnetic media is of significant interests for understanding of quantum phenomenon influencing the electronic states and processes, as well as giant and tunnelling magnetoresistive effects.

In our earlier publications $[10,11]$ we showed the results on the study of magnetic resonance phenomena and static magnetic properties of Fe-implanted PET. In this paper, we present the data of temperature dependence of conductance and magnetoresistive measurements and discuss magnetotransport properties of the composite Fe-PET layers.

\section{MATERIALS AND METHODOLOGY}

$40 \mu \mathrm{m}$ thick PET films were implanted by $\mathrm{Fe}^{+}$ions with energy of $40 \mathrm{keV}$ and fluences in the range of $(0.25-1.5) \times 10^{17} \mathrm{~cm}^{-2}$ at ion current density of $4 \mu \mathrm{A} / \mathrm{cm}^{2}$ in residual vacuum of $10^{-5}$ Torr. The sample holder was watercooled to prevent polymer overheating and thermal degradation during the implantation. To provide ohmic electrical contacts to the implanted layer, the electrodes were deposited on the ends of the samples using silver paste. Temperature dependence of resistance was measured using quasifour-probe method in the interval of $10-300 \mathrm{~K}$. The meas- 
urements were carried out at voltages corresponding to linear interval of current-voltage dependence. Effect of external magnetic field on charge carrier transport is studied for inplane configuration (magnetic field is parallel to the implanted layer and it is perpendicular to the direction of electric field or current) for two opposite field directions, i.e. from 0 to $5 \mathrm{~T}$ and from 0 to $-5 \mathrm{~T}$ at liquid helium temperature $(4.2 \mathrm{~K})$.

\section{RESULTS AND DISCUSSION}

It was shown in our earlier studies that the high-fluence implantation of iron into PET causes the nucleation of metal NPs in the near-surface layer [10]. The similar effect was also found for the case of $\mathrm{Co}^{+}$and $\mathrm{Fe}^{+}$ion implantation into polyimide [9, 13]. Implantation with moderate fluence of $0.75 \times 10^{17} \mathrm{~cm}^{-2}$ caused nucleation of metal inclusions which can have different shapes and can agglomerate forming elongated structures (see Fig. 1a). Lateral size of the Fe NPs and their spatial density were increased with fluence that finally leaded to the formation of quasi-continuous planar labyrinth-like structures as can be seen in Fig. (1c). The analysis of the electron diffraction patterns showed that there were $\alpha$-phase metallic Fe with the traces of non-magnetic iron oxides (mainly, $\mathrm{Fe}_{2} \mathrm{O}_{3}$ ), as well as graphite and amorphous carbon phases present in the implanted layers. The study of conductance and magnetisation as a function of ion fluence demonstrated a gradual increase of these parameters with a stepwise change at values above $(0.75-0.9) \times 10^{17} \mathrm{~cm}^{-2}$ and following saturation $[10,11]$. A so-called percolation transition in electrical and magnetic properties was suggested due to the formation of quasi-continuous granular iron layer in which close-located but non-overlapping Fe NPs or grains are situated in the heavily carbonized polymer.

For the current case we measured resistance of the implanted samples as a function of temperature. The absolute values for room and one of low temperatures are presented in Table 1. The samples implanted with fluences of $0.25,0.5$ and $0.75 \times 10^{17} \mathrm{~cm}^{-2}$ demonstrate monotonous decrease of resistance with temperature, i.e. a negative temperature coefficient of resistance, that represents a well-known behavior for ion-implanted polymers [8, 14]. One of these dependences is presented in Fig. (2). The other dependence presented in this figure corresponds to the sample implanted with fluences of $1.0 \times 10^{17} \mathrm{~cm}^{-2}$. It is obvious that the sample implanted with higher fluence has a positive temperature coefficient of resistance. Hence, the figure demonstrates the Andersen IMT with the fluence increase where one of the samples is on the "dielectric side" of the transition but the other one is on the "metallic side". The dominant mechanism of charge carrier transport in implanted polymer layers is typically described by VRH. The temperature dependence of conductance in this case is defined by well-known equation [14]

$$
\sigma(T)=\sigma_{0} \exp \left[-\left(T_{0} / T\right)^{\gamma}\right]
$$

For convenience, we converted this equation to resistance $R(T)=R_{0} \exp \left(T_{0} / T\right)^{\gamma}$,

where $\gamma$ is a power characterizing the dimension $(D)$ of hopping, $\gamma=1 /(1+D), D=1,2,3$. However, the attempts to fit the "dielectric" curve with one of the possible $\gamma=1 / 2,1 / 3$ or $1 / 4$ did not lead to any reasonable agreement. It has been shown earlier for the case of polyimide implanted by highfluence $\mathrm{Co}^{+}$ions that the presence of metal clusters in the carbonized by implantation polymer matrix caused complex temperature dependence of conductance that significantly deviated from eq. (1) [8]. For instance, quantum corrections to conductance due to a weak localisation of electrons and electron-electron interaction could be possible reasons [15, 16]. For the current case, as shown in insertion to Fig. (2), the dependence for the sample implanted with fluence of $0.75 \times 10^{17} \mathrm{~cm}^{-2}$ can be well approximated by a linear function in coordinates $\ln \left(R / R_{295}\right)-(1 / T)^{1 / 2}$ below $80 \mathrm{~K}$. Hence, it can be suggested that at high temperatures there are few possible mechanisms contributing into the charge carrier transport, while for the temperature interval below $80 \mathrm{~K}$ there is a dominant mechanism giving the $R \sim \exp (1 / T)^{1 / 2}$ dependence. It was shown elsewhere [17] that tunnelling of electrons can be such a mechanism in a disordered material, in particular, in granular metals. The other curve in Fig. (2) corresponds to metallic type of conductance. It should be mentioned that metal concentration in the implanted layer for the corresponding fluence is only about 20-25 at. \% [18], which is not enough for the physical percolation of metal inclusions. In this case the contribution into conductance from the radiation-induced carbonised phase of polymer must be considered [3-7].

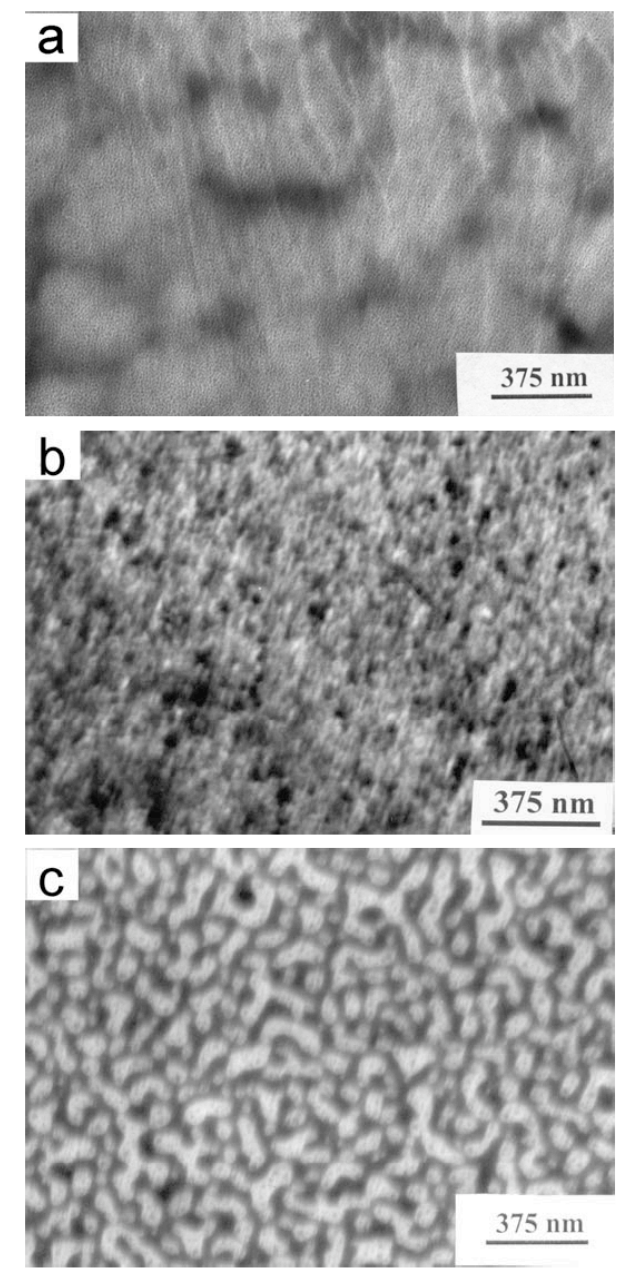

Fig. (1). TEM images of PET implanted with fluences of (a) 0.75 , (b) 1.25 and (c) $1.5 \times 10^{17} \mathrm{~cm}^{-2}$. Images (b) and (c) are reprinted with permission from [9]. Copyright 2006, Willey. 
Table 1. Dependance of Resistance of Samples on Implantation Fluence for Two Temperatures of Measurements

\begin{tabular}{|c|c|c|}
\hline Fluence, $\mathbf{c m}^{-2}$ & Resistance at 295 K, Ohm & Resistance at 15 K, Ohm \\
\hline \hline $2.5 \times 10^{16}$ & $1.98 \times 10^{7}$ & $1.08 \times 10^{8}$ \\
\hline $5.0 \times 10^{16}$ & $4.53 \times 10^{5}$ & $7.92 \times 10^{5}$ \\
\hline $7.5 \times 10^{16}$ & $6.17 \times 10^{4}$ & $1.12 \times 10^{3}$ \\
\hline $1.0 \times 10^{17}$ & $1.86 \times 10^{3}$ & $1.01 \times 10^{3}$ \\
\hline $1.5 \times 10^{17}$ & $1.23 \times 10^{3}$ & \\
\hline
\end{tabular}

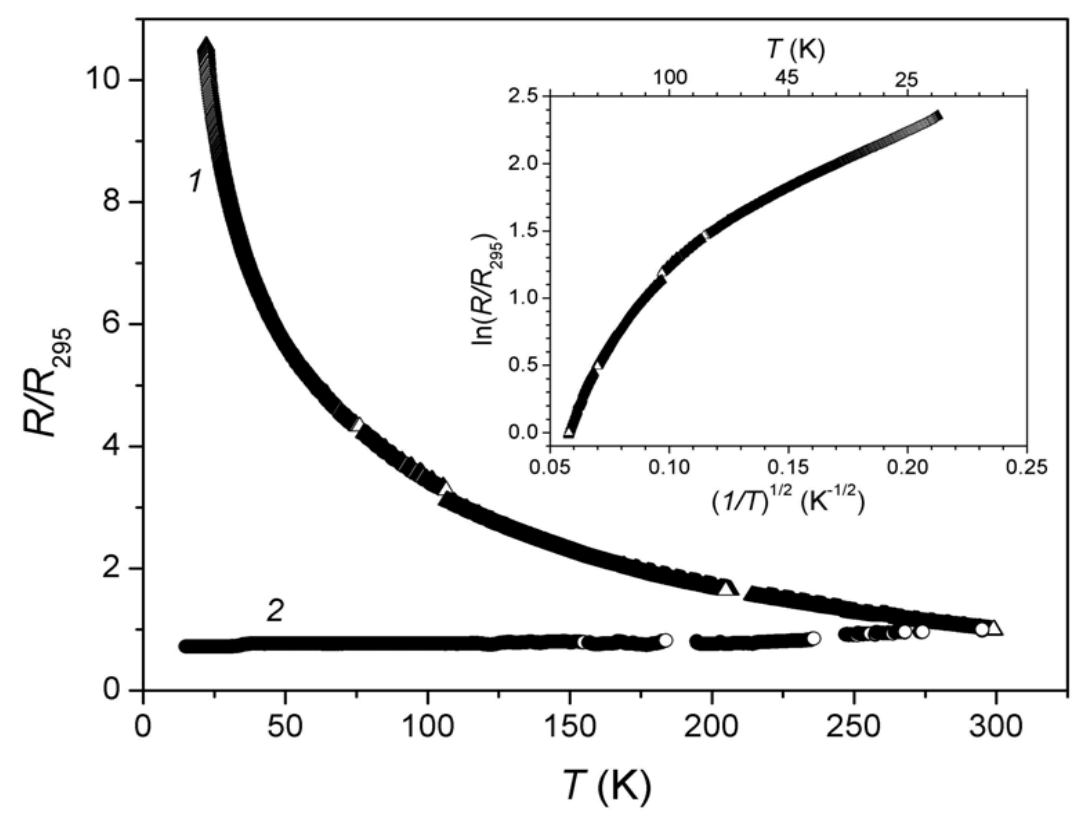

Fig. (2). Temperature dependence of relative resistance (with respect to room temperature value $R_{295}$ ) for PET implanted by Fe ${ }^{+}$ions with fluences of (1) 0.75 and (2) $1.0 \times 10^{17} \mathrm{~cm}^{-2}$. Dependence (1) is also presented in coordinates $\ln \left(R / R_{295}\right)-(1 / T)^{1 / 2}$ in insertion.

The samples implanted with low fluences (0.25-0.75 $\times 10^{17} \mathrm{~cm}^{-2}$ ), which are on the "dielectric side" of IMT, showed non-monotonous magnetoresistive dependences. One can see the dependence of relative resistance $(\Delta R=R-$ $R_{0}$, where $R$ and $R_{0}$ are the values in the presence and absence of the field, correspondingly) on the external magnetic field for the sample implanted with fluence of $0.75 \times 10^{17} \mathrm{~cm}^{-2}$ in Fig. (3). It demonstrates the decrease of resistance in an increasing field, i.e. negative sign of magnetoresistance. In general case, negative magnetoresistance can be explained by taking into account either interference processes for VRH [19] or approximation of effective medium [20]. In these cases, the dependence of resistance on external magnetic field is typically monotonous. However, one can see that our dependence has extremes. It has a strong minimum at about 70-80 $\mathrm{mT}$ (see insert to Fig. 3) and wider maximum at ca. 1 $\mathrm{T}$ for both directions of the scanning magnetic field. Our previous study showed that the sample implanted with fluence of $0.75 \times 10^{17} \mathrm{~cm}^{-2}$ had a coercive force of about $30 \mathrm{mT}$ at $4 \mathrm{~K}$ [11]. This value is different from the above-mentioned 70-80 $\mathrm{mT}$. For better understanding of the phenomenon, one should also mention the presence of so-called training effect, i.e. the minimum at 70-80 $\mathrm{mT}$ becomes blur under the repetition of increase of the external magnetic field from 0 to $5 \mathrm{~T}$.
However, it re-appears after the field scan in the opposite direction, i.e. from 0 to $-5 \mathrm{~T}$. Similar training effect and contribution of the domain wall scattering effect to magnetoresistance were observed elsewhere for the granular $\mathrm{CoFe}$ $\mathrm{AgCu}$ films when magnetic percolation took place at ferromagnetic concentrations much lower than the physical percolation threshold [12], i.e. in the case which is similar to ours. Thus, we can suggest that the observed difference between the values of coercive force (of ca. $30 \mathrm{mT}$ ) and the current extremes (of 70-80 $\mathrm{mT}$ ) is most probably caused by contribution of electron scattering on the domain walls moving (changing their location) in the applied magnetic field. Reason for the observed increase of relative resistance at about $1 \mathrm{~T}$ (Fig. 3) is not very clear. One of possible explanations can be a dominance of another mechanism of charge carrier transport (leading to lower conductance), for instance, the VRH through non-magnetic conductive clusters (radiation-induced carbonized phase of the polymer). The magnetotransport measurements of this sample at higher temperatures showed rapid increase in scattering of experimental points, and the relative change in magnetoresistance cannot be measured when the blocking temperature is reached, i.e. when the Fe NPs experience transition from the ferromagnetic to the superparamagnetic state [11]. 
In our case, the IMT transition takes place at fluences about $1.0 \times 10^{17} \mathrm{~cm}^{-2}$. However, as can be seen from the TEM image (Fig. 1b), the nanoparticles do not yet form a continuous granular layer. Nevertheless, the intermixture of the Fe NPs with the conductive carbonized phase provides conditions for the electrical percolation. From the above presented results on magnetoresistance measurements and the earlier obtained SQUID data for the same sample on the "dielectric side" of IMT [11] one can conclude that the magnetic percolation already occurs at lower implantation fluence of $0.75 \times 10^{17} \mathrm{~cm}^{-2}$. This sample demonstrated ferromagnetic properties up to $150 \mathrm{~K}$, the magnetization saturated at ca. 0.5 $\mathrm{T}$ and the in-plane coercive force reached $30 \mathrm{mT}$ at liquid helium temperatures [11]. This lower threshold fluence is most probably related to both direct dipole-dipole coupling of the iron NPs and indirect interactions through electrons of the carbonized polymer or individual iron atoms. Thus, despite the suggested tunnelling of electrons as the dominant mechanism of conductance in the absence of external magnetic field at low temperature, we cannot conclude about pure tunnelling magnetoresistive effect, for which the peaks in the magnetoresistive dependence must correspond to the value of coercive field $[21,22]$. In our case, the nonmonotonous magnetoresistive dependence can be explained by a complex interplay between (i) an anisotropic magnetoresistive (AMR) effect and (ii) a contribution from the scattering at the domain walls.

The magnetoresistive dependence for the sample implanted with fluence of $1.0 \times 10^{17} \mathrm{~cm}^{-2}$ is shown in Fig. (4).

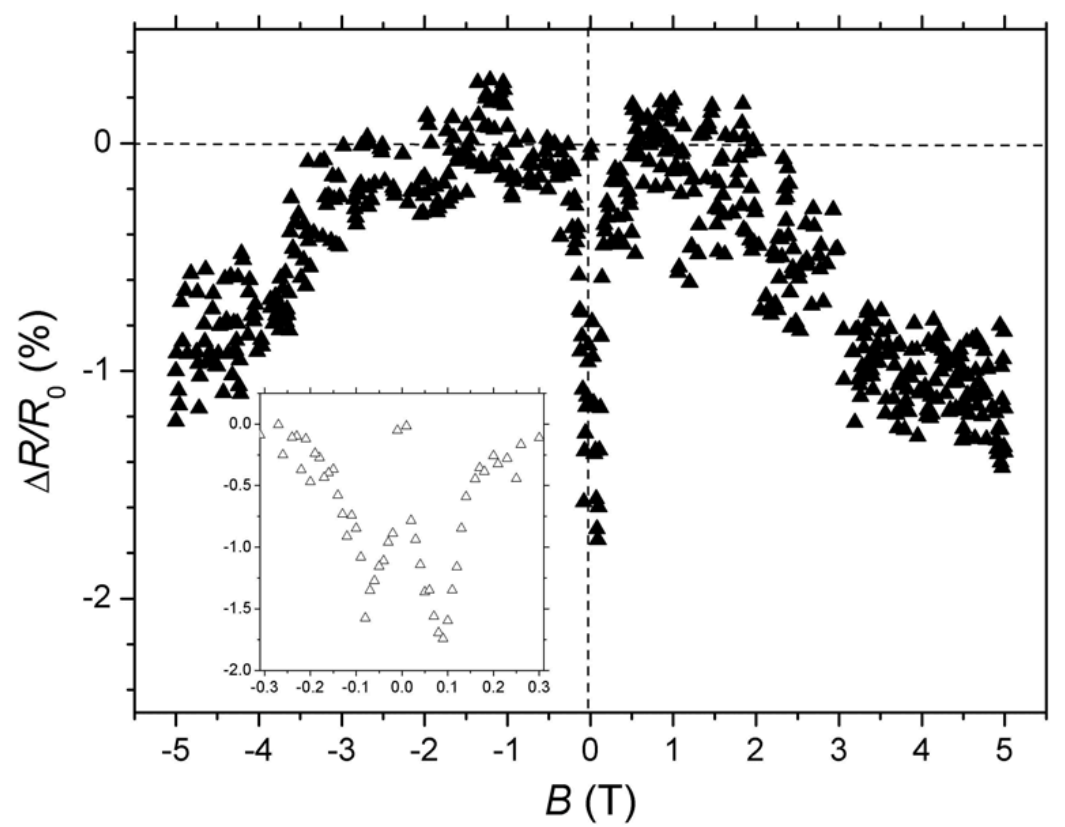

Fig. (3). Magnetoresistive dependence for PET implanted by $\mathrm{Fe}^{+}$ions with fluence of $0.75 \times 10^{17} \mathrm{~cm}^{-2}$. Insertion shows zoom into low field interval (up to $0.3 \mathrm{~T}$ ). Measurements are performed at $4.2 \mathrm{~K}$.

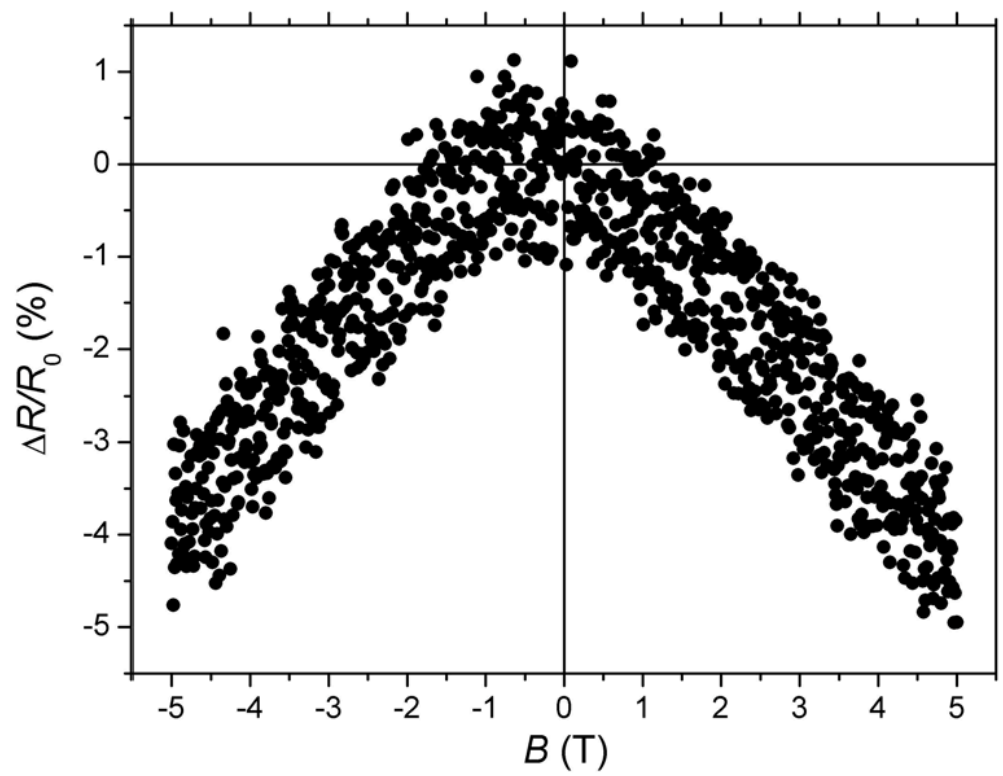

Fig. (4). Magnetoresistive dependence for PET implanted by $\mathrm{Fe}^{+}$ions with fluence of $1.0 \times 10^{17} \mathrm{~cm}^{-2}$. Measurements are performed at $4.2 \mathrm{~K}$. 
The relative resistance is found to be a monotonous decreasing function of the external magnetic field. Though the TEM data did not show the formation of continuous iron layer for this fluence yet [10], spatial density of the iron NPs is high enough to provide effective percolation conductivity and magnetic coupling. The observed dependence is similar to that assigned in some cases to ferromagnetic metals [23] and in some cases to granular ferromagnetic films. In the latter case the films typically show giant magnetoresistive (GMR) effect. However, there are no deviations which could be assigned to GMR for our sample. To explain it we can suggest a possibility of several contributions such as (i) ordinary magnetoresistance, (ii) GMR and (iii) AMR. The AMR can be a dominant component in particular due to severe radiation-damage and carbonized phase formation in the layer, where the iron NPs are located.

\section{CONCLUSIONS}

In conclusion, evolution of charge carrier transport mechanisms and magnetotransport properties is studied for the thin composite metal/polymer layers synthesized by lowenergy high-fluence implantation of $\mathrm{Fe}^{+}$ions into PET films. It has been shown that nucleation of the metals NPs and their coalescence with increase of the implantation fluence and iron concentration are crucial processes, governing the electrical and magnetic characteristics of the composites. It is found that for the samples implanted with relatively low fluences the most probable mechanisms of charge carrier transport are VRH and tunnelling of electrons. Increase of ion fluence up to $1.0 \times 10^{17} \mathrm{~cm}^{-2}$ leads to the formation of quasicontinuous granular iron layer in the carbonized polymer matrix that provides conditions for percolation and the insulator-to-metal transition in conductance. The magnetic percolation is found for lower fluence of $0.75 \times 10^{17} \mathrm{~cm}^{-2}$. These samples show negative magnetoresistance but it is not monotonous. Contributions from both the anisotropic magnetoresistance and the effect of charge carrier scattering at the domain walls should be taken into account for this transitional case.

\section{ACKNOWLEDGEMENTS}

One of the authors (V.N.P) acknowledges support of the Nanoparticle Platform at the Science Faculty of University of Gothenburg. Authors from Russia (R.I.K. and V.V.B.) would like to thank RAS Programme "New materials and structures".

\section{REFERENCES}

[1] Rück DM. Ion induced modification of polymers at energies between $100 \mathrm{keV}$ and $1 \mathrm{GeV}$ applied for optical waveguides and improved metal adhesion. Nucl Instrum Methods Phys Res B 2000; 166-167: 602-9.
[2] Khaibullin RI, Rameev BZ, Stepanov AL, et al. Ion beam synthesis of magnetic nanoparticles in polymers. In: Aktas B, Tagirov L, Mikailov F, Eds. Nanostructured magnetic materials and their applications. Dordrecht: Kluwer Academic 2004; pp. 33-54.

[3] Popok VN. Modification of surface layers of polymers by ion beams. Surf Investig 1999; 14: 843-59.

[4] Sviridov DV. Chemical aspects of implantation of high-energy ions into polymeric materials. Rus Chem Rev 2002; 71:315-27.

[5] Švorčik V, Rybka V, Miček I, et al. Structure and properties of polymers modified by ion implantation. Eur Polym J 1994; 30: 1411-5.

[6] Moliton A, Lucas B, Moreau C, Friend RH, Francois B. Ion implantation in conjugated polymers: mechanisms for generation of charge carriers. Philos Mag B 1994; 69: 1155-71.

[7] Marletta G, Iacona F. Chemical and physical property modifications induced by ion irradiation in polymers. In: Pauleau Y, Ed. Materials and processes for surface and interface engineering. NATO ASI Series, Series E, Appl Sci, V. 260. Dordrecht: Kluwer Academic 1995; pp. 597-640.

[8] Popok VN, Lukashevich MG, Lukashevich SM, Khaibullin RI, Bazarov VV. Charge carrier transport in polyimide with Co nanoparticles formed by ion implantation. Surf Sci 2004; 566-68: 327-31.

[9] Khaibullin RI, Popok VN, Bazarov VV, et al. Ion synthesis of iron granular films in polyimide. Nucl Instrum Methods Phys Res B 2002; 191: 810-4.

[10] Okay C, Rameev BZ, Khaibullin RI, et al. Ferromagnetic resonance study of iron implanted PET foils. Phys Stat Sol 2006(a); 203: 1525-32.

[11] Lukashevich MG, Batlle X, Labarta A, et al. Modification of magnetic properties of polyethyleneterephthalate by iron ion implantation. Nucl Instrum Methods Phys Res B 2007; 257: 589-92.

[12] Franko V, Battle X, Labarta A. Evidence of domain wall scattering in thin films of granular CoFe- $\mathrm{AgCu}$. Eur Phys J B 2000; 17: 4350 .

[13] Popok VN, Khaibullin RI, Toth A, Beshliu V, Hnatowicz V, Mackova A. Compositional alteration of polyimide under high fluence implantation by $\mathrm{Co}^{+}$and $\mathrm{Fe}^{+}$ions. Surf Sci 2003; 532: 1034-9.

[14] Wang Y, Bridwell LB, Giedd RE. Composite conduction in ionimplanted polymers. J Appl Phys 1993; 73: 474-6.

[15] Lee PA, Ramakrishan TV. Disordered electronic systems. Rev Mod Phys 1985; 57: 287-337.

[16] Altshuler BL, Aronov AG, Lee PA. Interaction effects in disordered Fermi systems in two dimensions. Phys Rev Lett 1980; 44: 1288-91.

[17] Sheng P, Abeles B, Arie Y. Hopping conductivity in granular metals. Phys Rev Lett 1973; 31: 44-7.

[18] Popok VN, Khaibullin RI, Bazarov VV, et al. Anomalous depth distribution of $\mathrm{Fe}$ and $\mathrm{Co}$ atoms in polyimide implanted to high fluence. Nucl Instrum Methods Phys Res B 2002; 191: 695-9.

[19] Schirmacher W. Quantum-interference magnetoconductivity in the variable-range-hopping regime. Phys Rev B 1990; 41: 24618 .

[20] Raikh ME, Wessels GF. Single-scattering-path approach to the negative magnetoresistance in the variable-range-hopping regime for two-dimensional electron systems. Phys Rev B 1993; 47: 15609

[21] Battle X, Labarta A. Finite-size effects in fine particles: magnetic and transport properties. J Phys D: Appl Phys 2002; 35: R1542.

[22] Holdenried M, Micklitz H. Tunneling magnetoresistance in granular films made of well-defined Co clusters embedded in an inertgas matrix. Eur Phys J B 2000; 13: 205-8.

[23] Smit S. Magnetoresistance of ferromagnetic metals and alloys at low temperatures. Physica 1951; 16: 612-27. 